\title{
The troublesome triad: festoons, malar mounds, and palpebral bags
}

\author{
Lam Kar Wai Phoebe, MD \\ Dr. Lam's Clinic, Kowloon, Hong Kong
}

\begin{abstract}
The ill-defined lid-cheek junction continues to be one of the most challenging regions for rejuvenation. Malar mounds, festoons or palpebral bags, may occur alone, or in combination, in the infraorbital area. These pathological variants are distinctly different and may contribute to patients' perception of their eyebags. Traditional lower blepharoplasty (fat repositioning/ removal) with skin adjustment alone may be insufficient in addressing all of these pathologies, and recurrence may occur. This literature review aims to look at current definitions and treatment of festoons, malar mounds, and palpebral bags in Asian blepharoplasty. A Medline literature search for articles with anatomic descriptions of malar mounds, palpebral bags and festoons, particularly in Asian eyelids, was performed. This was supplemented by a manual search of the references of articles obtained. The details of all of the different surgical options for eyelid rejuvenation are beyond the scope of this review article. Thus, only the basic concepts that illustrate the anatomical relationship and the natural progression of malar mounds, festoons, and palpebral bags will be discussed in this article. Some of the treatment options, both invasive and non-invasive, will also be reviewed. There is no singular treatment for malar mounds, festoons, and palpebral bags. Standard lower blepharoplasty with skin excision is ineffective to lift and re-drape malar mounds or festoons. Repositioning of the malar septum, on the other hand, allows for resolution of the edema (malar festoons) above its cutaneous insertion. A number of liposculpturing techniques to volumize deficient areas, such as fat repositioning (cheek, suborbicularis and descended malar fat pad) with lifting of the midface, can be beneficial to restore a smooth lid-cheek junction.Non-invasive techniques like laser resurfacing and Ultherapy ${ }^{\circledast}$ (Ulthera, Inc., Meza, AZ, USA) may be useful in periorbital rejuvenation in selected cases.
\end{abstract}

Keywords: festoon; malar mound; palpebral bag; orbitomalar septum; periorbital rejuvenation

\section{Introduction}

Malar mounds, palpebral bags and/or festoons may appear alone or in combination with each other in the infra-orbital area. Even though these occur within same region, there is a distinct difference between their three pathological variants, and all are likely to fall within a continuum of anatomic findings (Fig. 1 and Table 1) [1].

Standard lower bleopharoplasty is one of the treatment modalities for what is colloquially termed as "Asian eye bags."
This, however, is insufficient to address all of these anatomical changes and to restore a youthful smooth lid cheek junction. Furthermore, most elderly Asian patients are not interested in a complicated surgical procedure for periorbital rejuvenation, particularly if it is uncomfortable to go through with a long period of recovery time [2].

Articles in English with anatomic descriptions of malar mounds and festoons, particularly in Asian patients and in lower blepharoplasty procedures, as well as details of corrective procedures and methods of periorbital rejuvenation were iden- 
tified from the OVID Medline database (1966-2009).

Details of the many different surgical options for eyelid rejuvenation are beyond the scope of this review article. However, some of the basic concepts that illustrate the relationship between anatomic contribution of malar mounds, festoons and palpebral bags and their natural progression, together with some of the more contemporary procedures, which are generally acceptable to patients, will be discussed in this article.

\section{Variation in Asian lower eyelids}

There is no article that discusses the overall incidence, prevalence and management of festoons and malar mounds in Asians that can be found, yet, some intrinsic anatomical struc-

A

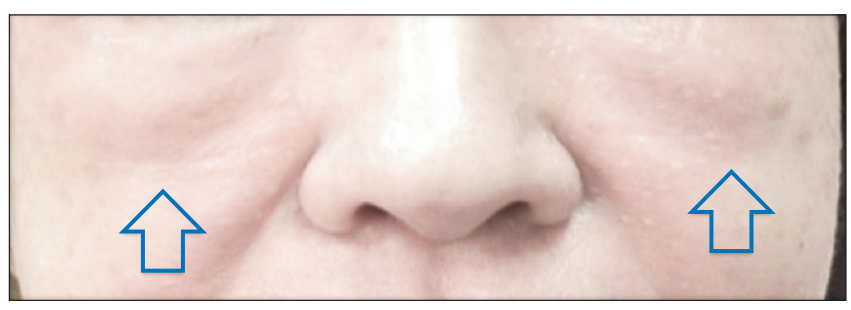

B

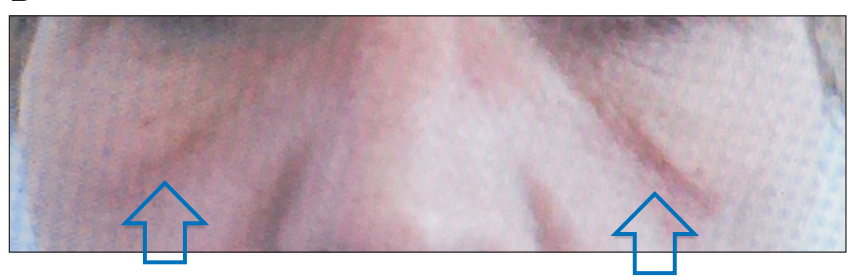

C

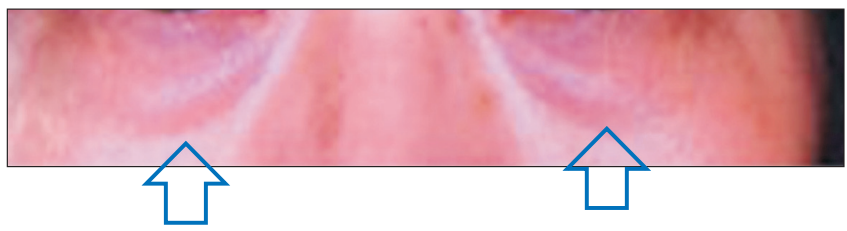

Fig. 1. The morphological appearance of the three pathological variants, (A) Malar mound, (B) festoon, (C) palpebral bag in the infraorbital region. tural differences amongst Asian eyelids have been identified and discussed in recent studies [3-9].

It was observed that, morphologically, Asians in general have puffier and fuller lower eyelids than do other races. Anatomically, they have high levels of anterior and superior orbital fat and a higher fusional location of the orbital septum with capsulopalpebral fascia (CPF) as compared with Caucasians, in which fibers of their CPF are sparse among the subcutaneous tissue. Other anatomical variations identified in a histological microscopic analysis (e.g. superior extended orbital fat) were found to be attached to the inferior tarsal border in Chinese and Korean eyes, but not in Japanese eyes $[4,6,10]$.

\section{Festoons and malar mounds}

A "mound" is an elevation, and the term "festoon" describes the hanging of tissues between two points [1].

Festoons and malar mounds are bounded by the insertion of the orbitomalar septum, which extends caudally in the lateral half of the lid-cheek junction, and then curves upward and lateral to the outer canthus. This also outlines the caudal margin of an elliptical portion of the superior lateral cheek fat [11]. The orbitomalar septum (Fig. 2) acts as an impermeable membrane. Tissue edema can accumulate above the cutaneous insertion of this septum, and below the orbital rim, where the facial network originates [12].

This should be distinguished from the protrusion of orbital fat against the weakened orbital septum or orbicularis muscle in the case of palpebral bags, in which the infraorbital fat is instead separated by the arcuate expansion of the inferior oblique laterally, and the valley of the inferior oblique medially and eyelid fluid has an even contour that does not respect the orbital compartments in its distribution [13].

Eyelid fluid does not change much in up-gaze and downgaze, whereas bulging of the lower lid orbital fat may be at least temporarily reduced by contraction of the orbicularis muscle due to its enhanced support. Additionally, eyelid fluid moves and gathers below the orbital rim as we press on the orbital rim,

Table 1. Features and characteristics of the three pathological variants (Malar mound [MM]; orbicularis oculi muscle [OOM]; orbitomalar septum [OrbS])

\begin{tabular}{|c|c|c|c|}
\hline & MM & Festoon & Palpebral bag \\
\hline Description & An "Elevation" & Hanging of tissues between 2 points & EYE BAG **Compartmentalized fat \\
\hline Composition & FAT insinuate within OOM & $\begin{array}{l}\text { Purely of skin and OOM: } \\
\text { accumulation of FAT or FLUID }\end{array}$ & $\begin{array}{l}\text { Protrusion of orbital FAT } \\
\text { against weakened OrbS or OOM }\end{array}$ \\
\hline Boundary & \multicolumn{2}{|c|}{ Insertion of OrbS } & Inferior orbital rim \\
\hline Relationship with OrbS & \multicolumn{2}{|c|}{ In FRONT of OrbS } & BEHIND of OrbS \\
\hline
\end{tabular}




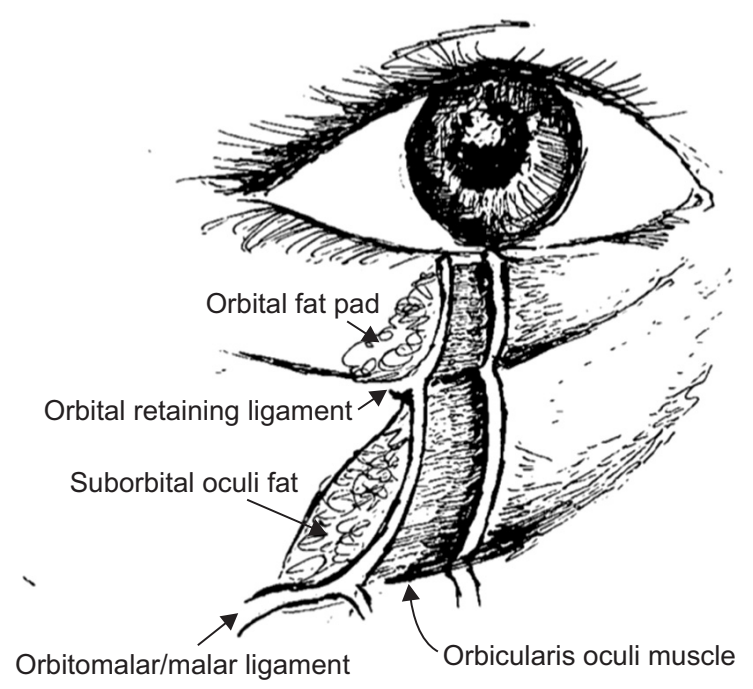

Fig. 2. The relationship between the orbitomalar septum, lid-cheek junction, orbital and suborbital oculi fat.

whereas infraorbital fat does not move [13].

Festoons are composed purely of skin and the orbicularis oculi muscle that can accumulate fat or fluid, forming edematous sacks that bulge from the area of malar eminence [1]. They develop from a progressive distribution of the skin and muscle secondary to chronic malar edema. Festoons are usually found in the following areas: the pre-septal, orbital, and jugal regions of the lower lid [14]. It has been reported that orbicularis oculi tonicity may facilitate lymphatic flow, and that the loss of muscle tone (e.g. after surgery, botox injection) in this area can result in edematous festoons [1]. Other exacerbating factors include a salty meal (due to increased sodium intake), systemic edematous state (e.g. chronic renal disease, allergy, or hepatic cirrhosis), or drug use [1,13].

With time, the orbicularis muscle attenuates and progressively sags until folds of muscle are suspended across the lower lid. If the orbital septum is also lax, the septum may also sag and invaginate into the upper part of skin-muscle, adding a septal pouch to the complex. The festoons typically disappear when the muscle contracts, and reappear as the muscle relaxes [14,15].

Malar mounds develop as the fat insinuates into the grooves and fenestrations in the orbicularis oculi muscle. This is more common in patients older than 50 years of age $[13,15]$. Gravity seems to have little effect on the development of malar mounds. However, if the swelling in the malar mound region is primarily subdermal, then the presentation is known as malar festoons instead. These "malar bags" may also atrophy with age, resulting in the appearance of "festoons" of lax infraorbital malar skin that hang over the fixed line of orbitomalar septal insertion $[11,14]$.

Hoenig et al.[14] observed that malar festoons appear to be correctable only if the malar septum is affected. The authors reported that by vertically elevating the soft tissue of the cheek (i.e. vertical subperiosteal mid-face lift) and repositioning the malar septum, the tissue edema (i.e. malar festoons) above its cutaneous insertion resolves. This may be done in combination with the repositioning of the descended suborbicularis oculi fat (SOOF) and malar fat pad, done by anchoring it to the temporalis fascia to provide a superotemporal lift in order to restore the natural contours of the eyelid and cheek region $[1,14]$. The authors identified that the subset of patients with a flat malar eminence or lack of youthful malar convexity are anatomically predisposed to prolonged postoperative edema and recurrence [1].

Rosenberg et al. proposed the modification of the fatty component of malar bags with suction lipectomy (i.e. liposuction of subdermal fat and edema), but did not give any consideration of the orbicularis muscle [16]. As such, this approach does not address muscular or ligamentous attenuation and is most likely only to benefit a subset of patients with primarily a fat and/or fluid collection $[1,16]$.

Caudal to the orbitomalar septum, the malar fat pad is supported in its youthful location by multiple fibroelastic fascial septa that extend through the fatty cheek mass originating from the underlying superficial fascia, which invests the mimetic muscles to insert into the overlying cutaneous dermis. Chronic recurrent tissue expansion of the skin of the lower anterior cheek, if combined with stretching of the supporting infraorbital fascial septa, results in downward migration of the malar fat pad [11].

Restoration of midface volume can improve a saggy appearance secondary to infraorbital and cheek fat atrophy. Additionally, it effaces folds and depressions, provides support to adjacent areas and disguises physical descent of midface structures. Volumizing deficient areas can be achieved via the transposition of orbital fat pedicles or via autologous fat grafting to volume-deficient sites. Alternative options include injectables (e.g. non-permanent fillers like hyaluronic acid filler), poly L-lactic acid, or solid synthetic implants, which allow for augmentation of facial framework [17-21].

Older patients may also benefit from procedures directed at skin adjustment (e.g. SMAS lifting and/or tightening and repositioning of the orbicularis oculi muscle). A number of modifications in the use of myocutaneous skin flaps in the restoring of a 
smooth lid cheek junction have been described [6,15,22-24].

Fractional laser resurfacing has been demonstrated to improve the appearance of malar bags and rejuvenate eyelid skin and its use has been reported with some success and without the risks and complications associated with non-fractional devices $[12,22,25]$. However, these techniques do not reposition the descended structures. It is unclear exactly how they modify local edema and adipose accumulations, but through collagen stimulation and soft tissue tightening, they may correct malar skinfolds or reduce malar prominence. Furthermore, scarring ectropion, and post-inflammatory hyperpigmentation may possibly occur following these treatments [14,20-24].

\section{Palpebral bags}

Palpebral bags can develop at an early age. They occur as the infra-orbital fat bulges outward against an attenuated or weak orbital septum and oculi muscle of the lower eyelid. Reinforcement of both the attenuated orbital septum and oculi muscle appears to be an important aspect not to neglect in the correction of palpebral bags $[21,26,27]$. Eyelid vector should be taken into consideration when planning surgery. In circumstances in which the tip of the cornea (i.e. globe prominence) is more anterior than the prominence of the lower lid/suborbital area (cheek), a "negative-vector" (Fig. 3) is present. Special care must be taken when approaching these cases, as lid over-tightening can bowstring the globe, and excess skin excision may lead to a higher incidence of lower lid malposition [28].

Tear trough (Fig. 4) often relates to the underlying bony structure and is particularly associated with age-related maxillary hypoplasia, in combination with the loss of subcutaneous fat with thinning of the skin over the orbital rim ligaments and cheek descent [13]. There is still controversy as to whether treatment for the herniation of fat in palpebral bags is best-determined, together with the presence and severity of a tear trough deformity or without the consideration of tear-trough deformity.

Whilst over-resection of infra-orbital fat may replace the original bulge deformity to an iatrogenic focal hollow, under-resection may require an additional surgery to smooth out the lid. Therefore, one need to assess the balance between the difference between the infra-orbital volume deficit and the native fat available for preservation, the native fat can be either excised, repositioned, or a combination of the two.

There have been a number of modifications made to the "intra-SOOF" procedure whereby the fat pads are temporarily secured internally or externally with matrix sutures through cheek skin $[2,29]$. There are centers which support the use of lower eyelid fat repositioning alone; others may combine lower eyelid

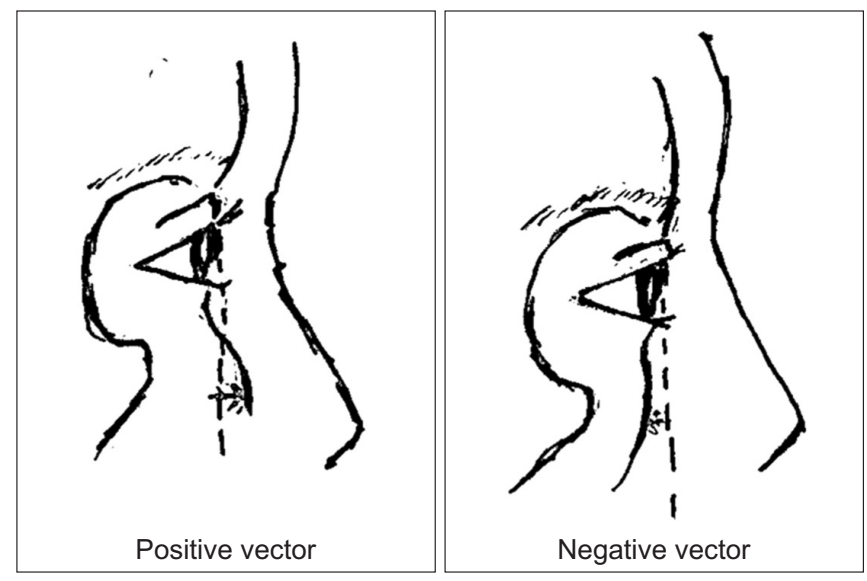

Fig. 3. Anatomical difference between positive and negative vectors, illustrating the relationship between the tip of the cornea and cheek.

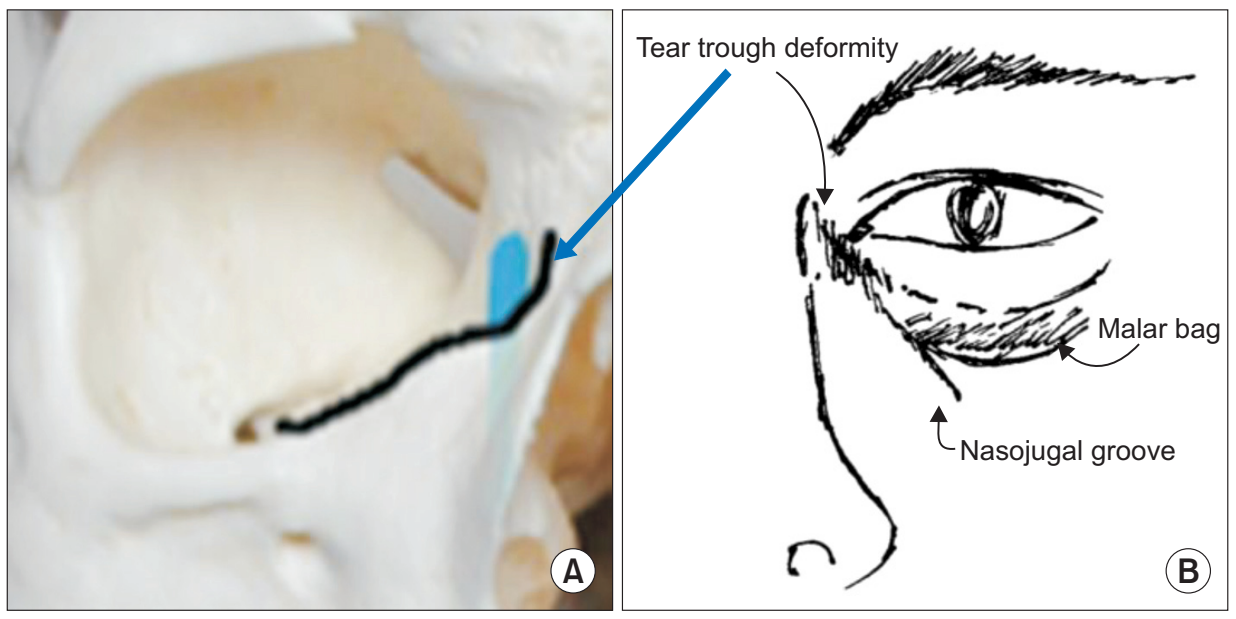

Fig. 4. The relationship between (A) Tear trough and underlying orbital bone rim and (B) Anatomical appearance of tear trough deformity. 
fat repositioning with a mid-face lift, regardless of the condition of the tear-trough $[2,13,30]$. The utilization of fat repositioning (i.e. manipulation of the septal fat pads without septal reset) is preferred to fat mobilization (i.e. arcus release and septal release) because in the latter, there is a risk of middle lamellar contracture with mobilization [30].

In circumstances in which the volume deficit is greater than that of the native fat available for preservation, then CPF repair (in which the inferior portion of the CPF is sutured to the arcus marginalis with interrupted 5-0 absorbable sutures, reinforcing the overlying septum) alone, or in combination with other procedures mentioned above, for volumizing deficient sites e.g. autologous fat grafting, fillers or facial implants), is an option [4].

Aging of the skin is histologically characterized by the loss of dermal elastic fibers that are gradually replaced by collagen of a fibrotic nature. Thus, alterations in dermal elasticity have been shown to be the main culprit that leads to the changes in appearance that occur with facial aging [31].

Rejuvenation of the lower eyelid skin is complex, but can be accomplished in a stepwise approach using a combination of procedures. Only some of the more effective lower eyelid rejuvenation treatments are discussed in this review.

Trichloroacetic acid (TCA) $30 \%$ peel is an ablative skin rejuvenation, which is effective in the managing of dermatochalasis of the lower eyelid. However, the typical results achieved following a TCA $30 \%$ peel are not as dramatic as the results of a laser procedure, but recovery is usually less complicated after the former $[17,29]$.

Fractionated resurfacing produces microcolumns of laser penetration while leaving intact skin bridges. This technology may be beneficial for younger patients as a solitary skin tightening procedure, or with multiple treatments in more aged skin. Both fractionated $\mathrm{CO}_{2}$ lasers and fractionated 1550-nm erbiumdoped technology has been reported to produce significant tightening of the eyelids [29]. However, fractional resurfacing can only restore lower eyelid skin tone and reduce fine wrinkles, and mainly serves to correct skin laxity. Laser resurfacing cannot correct or reposition prolapse of orbital fat or treat palpebral bulging. Skin damage and post-inflammatory hyperpigmentation can occur and are the most important adverse effects to note for in Asian populations [32].

The thickness of the lower eyelid epidermis is usually $0.5-0.6$ $\mathrm{mm}$, but only $0.2 \mathrm{~mm}$ in some patients, and that of the orbicularis muscle is $1.7-2.2 \mathrm{~mm}[33,34]$. The $3 \mathrm{~mm}$ transducer of Ultherapy ${ }^{\circledR}$ (Ulthera, Inc., Meza, AZ, USA) (HIFU, high intensity focal ultrasound) can treat both deep dermis and superficial muscular aponeurotic characteristics, sparing the dermis, and the $1.5 \mathrm{~mm}$ transducer can coagulate the lower epidermis and dermal layer [32]. There is evidence to suggest that the thermal effect of Ultherapy ${ }^{\circledR}$ (Ulthera, Inc., Meza, AZ, USA) (HIFU) can tighten the orbicularis muscle, orbital septum and intra-orbital fat, which therefore can be beneficial as a non-invasive rejuvenation technique to restore the shape of the lower palpebral bag in a subset of Asian patients. These are young patients $(<50$ years old), whom have never had previous surgery or treatment at the lower orbital rim (e.g. face-lift, soft tissue augmentation, medium depth peels and/or dermal photo-rejuvenation) [32].

A recent advancement in peri-orbital rejuvenation is the addition of a noninvasive polymer emulsion. This can be applied to the under-eye area with resultant in-situ cross-linking functionality, and formation of an invisible film. Preliminary study had identified a one-grade improvement in lower lid bag protrusion and increased epidermal thickness following use of this method [31].

\section{Discussion}

Traditional blepharoplasty and conservative fat removal still plays a role for patients with palpebral bags and prominent orbital fat. Fat repositioning (i.e. subperiosteally) through a transconjunctival approach is an option for patients with adequate orbital fat and a significant tear trough depression. However, it cannot correct malar loops with muscle pouches, or sagging of the lower orbicular muscles (ptosis). Even with reinforcement of the lower part of the orbicularis muscle, it is ineffective to lift and re-drape malar mounds or festoons $[15,25]$. The repositioning of the malar septum, on the other hand, allows for resolution of the edema (malar festoons) above its cutaneous insertion [14].

Over or under-resection of infra-orbital fat in patients with palpebral bags is unsatisfactory and may require additional surgery to smooth out the lid-cheek junction [13]. The eyelid vector should be taken into consideration prior to surgery to avoid post-operative lid malposition.

The use of CPF repair, or the combination of fat repositioning with elevation of the midface cheek fat (SOOF and descended malar fat pad) and/ or lipo-sculpturing to volume deficient areas, especially in older patients, can be beneficial to restore a smooth lid-cheek junction, regardless of tear trough deformity in some patients $[2,13,30,35,36]$.

When there is maxillary hypoplasia, substantial deflation or descent of the malar and periorbital tissues, SMAS lift and/ 
or tightening or a periorbital implant can be considered. Prolonged postoperative edema and recurrence may be expected in these patients.

Non-invasive techniques (e.g. laser resurfacing and Ultherapy $^{\circledR}$ [Ulthera, Inc., Meza, AZ, USA]) may be useful in selected cases as an adjunct for periorbital rejuvenation [32]. New advances in technology, such as noninvasive polymer emulsion, may receive use in the future.

\section{Conclusion}

The importance of awareness of the existence of festoons, malar mounds and palpebral bags and the interpretation of the relative contribution of these various anatomic components is vital to success following the planning of treatment for patients.

Preoperative assessment and discussion with interested patients is important to identify these variants, and understanding the patient's perceptions and expectations is equally important, so that the proposed aesthetic plan and result becomes more acceptable.

A combination of malar septum repositioning, midface lifting and liposculpturing techniques targeted at individual abnormalities will facilitate resolution of the "troublesome triad" and restore a youthful lid cheek complex. Ongoing scientific advances will continue to expand our treatment options for periorbital rejuvenation.

\section{Conflicts of interest}

The author has nothing to disclose.

\section{References}

1. Kotlus BS, Schwarcz R. Malar festoons: anatomy and treatment strategies. Am J Cosmet Surg 2010;27:1-10.

2. Chuang AC, Lian SL. Periorbital rejuvenation surgery in the geriatric population. Int J Gerontol 2010;4:107-14.

3. Amrith S. Oriental eyelids--anatomical and surgical considerations. Singapore Med J 1991;32:316-8.

4. Carruthers JD, Carruthers A. Facial sculpting and tissue augmentation. Dermatol Surg 2005;31:1604-12.

5. Doxanas MT, Anderson RL. Oriental eyelids. An anatomic study. Arch Ophthalmol 1984;102:1232-5.

6. Kakizaki H, Jinsong Z, Zako M, Nakano T, Asamoto K, Miyaishi $\mathrm{O}$, et al. Microscopic anatomy of Asian lower eyelids. Ophthal Plast Reconstr Surg 2006;22:430-3.
7. Sayoc BT. Surgery of the oriental eyelid. Clin Plast Surg 1974;1:157-71.

8. Weingarten CZ. Blepharoplasty in the oriental eye. Trans Sect Otolaryngol Am Acad Ophthalmol Otolaryngol 1976;82:ORL 442-6.

9. Zubiri JS. Correction of the oriental eyelid. Clin Plast Surg 1981;8;725-37.

10. Cho WK, Paik JS, Han SH, Yang SW. Microscopic characteristics of lower eyelid retractors in Koreans. Korean J Ophthalmol 2011;25:344-8.

11. Owsley JQ, Roberts CL. Some anatomical observations on midface aging and long-term results of surgical treatment. Plast Reconstr Surg 2008;121:258-68.

12. Pessa JE, Garza JR. The malar septum: the anatomic basis of malar mounds and malar edema. Aesthet Surg J 1997;17:11-7.

13. Goldberg RA, McCann JD, Fiaschetti D, Ben Simon GJ. What causes eyelid bags? Analysis of 114 consecutive patients. Plast Reconstr Surg 2005;115:1395-402.

14. Hoenig JF, Knutti D, de la Fuente A. Vertical subperiosteal mid- face-lift for treatment of malar festoons. Aesthetic Plast Surg 2011;35:522-9.

15. Furnas DW. Festoons, mounds, and bags of the eyelids and cheek. Clin Plast Surg 1993;20:367-85.

16. Rosenberg GJ. Correction of saddlebag deformity of the lower eyelids by superficial suction lipectomy. Plast Reconstr Surg 1995;96:1061-5.

17. Carter SR, Seiff SR, Grant PE, Vigneron DB. The asian lower eyelid: a comparative anatomic study using high-resolution magnetic resonance imaging. Ophthal Plast Reconstr Surg 1998;14:227-34

18. Flowers RS. Tear trough implants for correction of tear trough deformity. Clin Plast Surg 1993;20:403-15.

19. Goldberg RA, Edelstein C, Shorr N. Fat repositioning in lower blepharoplasty to maintain infraorbital rim contour. Facial Plast Surg 1999;15:225-9.

20. Kranendonk S, Obagi S. Autologous fat transfer for periorbital rejuvenation: indications, technique, and complications. Dermatol Surg 2007;33:572-8.

21. Seiff SR. The fat pearl graft in ophthalmic plastic surgery: everyone wants to be a donor! Orbit 2002;21:105-9.

22. Farrior RT, Kassir RR. Management of malar folds in blepharoplasty. Laryngoscope 1998;108:1659-63.

23. Kahana A, Lucarelli MJ. Adjunctive transcanthotomy lateral suborbicularis fat lift and orbitomalar ligament resuspension in lower eyelid ectropion repair. Ophthal Plast Reconstr Surg 2009;25:1-6 
24. Roberts TL 3rd. Laser blepharoplasty and laser resurfacing of the periorbital area. Clin Plast Surg 1998;25:95-108.

25. Glasgold M, Lam SM, Glasgold R. Volumetric rejuvenation of the periorbital region. Facial Plast Surg 2010;26:252-9.

26. Hoenig JA, Shorr N, Goldberg RA. The versatile SOOF lift in oculoplastic surgery. Facial Plast Surg Clin North Am 1998;6:205-19.

27. De la Plaza R, Arroyo JM. A new technique for the treatment of palpebral bags. Plast Reconstr Surg 1998;81:677-87.

28. Massry GG. Comprehensive lower eyelid rejuvenation. Facial Plast Surg 2010:26:209-21.

29. Niamtu J. Surgical treatment options for lower eyelid aging. Cosmet Dermatol 2008;21:652-7.

30. Ross GL, Whitby DJ. Trends in aesthetic facial surgery: the hamra lower lid bleopharoplasty [Internet]. Gary Ross; Available from: http://www.garylross.com/trends-in-aesthetic- facial-surgery-the-hamra-lower-lid-blepharoplasty/.

31. Pilkington SJ, Belden S, Miller RA. The tricky tear trough: a review of topical cosmeceuticals for periorbital skin rejuvenation. J Clin Aesthet Dermatol 2015;8:39-47.

32. Pak CS, Lee YK, Jeong JH, Kim JH, Seo JD, Heo CY. Safety and efficacy of ulthera in the rejuvenation of aging lower eyelids: a pivotal clinical trial. Aesthetic Plast Surg 2014;38:861-8.

33. Lee Y, Hwang K. Skin thickness of Korean adults. Surg Radiol Anat 2002;24:183-9.

34. Bucay VW, Day D. Adjunctive skin care of the brow and periorbital region. Clin Plast Surg 2013;40:225-36.

35. Mendelson BC. Herniated fat and the orbital septum of the lower lid. Clin Plast Surg 1993;20:323-30.

36. Small RG. Extended lower eyelid blepharoplasty. Arch Ophthalmol 1981;99:1402-5. 